\title{
GENERALIZED CANAVATI TYPE $g$-FRACTIONAL POLYA TYPE INEQUALITIES
}

\author{
George A. Anastassiou \\ Department of Mathematical Sciences \\ University of Memphis \\ Memphis, TN 38152, USA
}

\begin{abstract}
We present here generalized Canavati type $g$-fractional Polya type inequalities. We cover also the iterated case. Our inequalities are with respect to all $L_{p}$ norms: $1 \leq p \leq \infty$. We finish with applications.
\end{abstract}

AMS Subject Classification: 26A33, 26D10, 26D15

Key Words: Polya inequality, fractional inequality, generalized fractional derivative, iterated generalized fractional derivative

\section{Introduction}

We are motivated by the following famous Polya's integral inequality, see [11], $[12$, p. 62$],[13]$ and $[14$, p. 83$]$.

Theorem 1. Let $f(x)$ be differentiable and not identically a constant on $[a, b]$ with $f(a)=f(b)=0$. Then there exists at least one point $\xi \in[a, b]$ such that

$$
\left|f^{\prime}(\xi)\right|>\frac{4}{(b-a)^{2}} \int_{a}^{b} f(x) d x .
$$

We need the following fractional calculus background:

Let $\alpha>0, m=[\alpha],[\cdot]$ is the integral part, $\beta=\alpha-m, 0<\beta<1$,

Received: October 15, 2019

(C) 2019 Academic Publications 
$f \in C([a, b]),[a, b] \subset \mathbb{R}, x \in[a, b]$. The gamma function $\Gamma$ is given by $\Gamma(\alpha)=$ $\int_{0}^{\infty} e^{-t} t^{\alpha-1} d t$. We define the left Riemann-Liouville integral

$$
\left(J_{\alpha}^{a+} f\right)(x)=\frac{1}{\Gamma(\alpha)} \int_{a}^{x}(x-t)^{\alpha-1} f(t) d t,
$$

$a \leq x \leq b$. We define the subspace $C_{a+}^{\alpha}([a, b])$ of $C^{m}([a, b])$ :

$$
C_{a+}^{\alpha}([a, b])=\left\{f \in C^{m}([a, b]): J_{1-\beta}^{a+} f^{(m)} \in C^{1}([a, b])\right\} .
$$

For $f \in C_{a+}^{\alpha}([a, b])$, we define the left generalized $\alpha$-fractional derivative of $f$ over $[a, b]$ as

$$
D_{a+}^{\alpha} f:=\left(J_{1-\beta}^{a+} f^{(m)}\right)^{\prime},
$$

see [1], p. 24. Canavati first in [6] introduced the above over $[0,1]$.

Notice that $D_{a+}^{\alpha} f \in C([a, b])$.

Furthermore we need:

Let again $\alpha>0, m=[\alpha], \beta=\alpha-m, f \in C([a, b])$, call the right RiemannLiouville fractional integral operator by

$$
\left(J_{b-}^{\alpha} f\right)(x):=\frac{1}{\Gamma(\alpha)} \int_{x}^{b}(t-x)^{\alpha-1} f(t) d t,
$$

$x \in[a, b]$, see also [7], [8], [9], [15], [2]. Define the subspace of functions

$$
C_{b-}^{\alpha}([a, b])=\left\{f \in C^{m}([a, b]): J_{b-}^{1-\beta} f^{(m)} \in C^{1}([a, b])\right\} .
$$

Define the right generalized $\alpha$-fractional derivative of $f$ over $[a, b]$ as

$$
D_{b-}^{\alpha} f=(-1)^{m-1}\left(J_{b-}^{1-\beta} f^{(m)}\right)^{\prime},
$$

see [2]. We set $D_{b-}^{0} f=f$. Notice that $D_{b-}^{\alpha} f \in C([a, b])$.

We are also motivated by the following fractional Polya type integral inequality without any boundary conditions.

Theorem 2. ([4], pp. 1-7) Let $0<\alpha<1, f \in C([a, b])$. Assume $f \in C_{a+}^{\alpha}\left(\left[a, \frac{a+b}{2}\right]\right)$ and $f \in C_{b-}^{\alpha}\left(\left[\frac{a+b}{2}, b\right]\right)$. Set

$$
M(f)=\max \left\{\left\|D_{a+}^{\alpha} f\right\|_{\infty,\left[a, \frac{a+b}{2}\right]},\left\|D_{b-}^{\alpha} f\right\|_{\infty,\left[\frac{a+b}{2}, b\right]}\right\} .
$$


Then

$$
\left|\int_{a}^{b} f(x) d x\right| \leq \int_{a}^{b}|f(x)| d x \leq M(f) \frac{(b-a)^{\alpha+1}}{\Gamma(\alpha+2) 2^{\alpha}} .
$$

Inequality (9) is sharp, namely it is attained by

$$
f_{*}(x)=\left\{\begin{array}{cl}
(x-a)^{\alpha}, & x \in\left[a, \frac{a+b}{2}\right] \\
(b-x)^{\alpha}, & x \in\left[\frac{a+b}{2}, b\right]
\end{array}\right\}, \quad 0<\alpha<1 .
$$

Clearly here non zero constant functions $f$ are excluded.

In this article we present very general $g$-fractional Polya type inequalities.

\section{Background}

Here we follow [5].

Let $g:[a, b] \rightarrow \mathbb{R}$ be a strictly increasing function. Let $f \in C^{n}([a, b])$, $n \in \mathbb{N}$. Assume that $g \in C^{1}([a, b])$, and $g^{-1} \in C^{n}([g(a), g(b)])$. Call $l:=f \circ g^{-1}:[g(a), g(b)] \rightarrow \mathbb{R}$. It is clear that $l, l^{\prime}, \ldots, l^{(n)}$ are continuous functions from $[g(a), g(b)]$ into $f([a, b]) \subseteq \mathbb{R}$.

Let $\nu \geq 1$ such that $[\nu]=n, n \in \mathbb{N}$ as above, where $[\cdot]$ is the integral part of the number.

Clearly when $0<\nu<1,[\nu]=0$ and $n=0$. Next we follow [1], pp. 7-9.

I) Let $h \in C([g(a), g(b)])$, we define the left Riemann-Liouville fractional integral as

$$
\left(J_{\nu}^{z_{0}} h\right)(z):=\frac{1}{\Gamma(\nu)} \int_{z_{0}}^{z}(z-t)^{\nu-1} h(t) d t,
$$

for $g(a) \leq z_{0} \leq z \leq g(b)$, where $\Gamma$ is the gamma function; $\Gamma(\nu)=\int_{0}^{\infty} e^{-t} t^{\nu-1} d t$.

We set $J_{0}^{z_{0}} h=h$.

Let $\alpha:=\nu-[\nu](0<\alpha<1)$. We define the subspace $C_{g\left(x_{0}\right)}^{\nu}([g(a), g(b)])$ of $C^{[\nu]}([g(a), g(b)])$, where $x_{0} \in[a, b]$ :

$$
\begin{gathered}
C_{g\left(x_{0}\right)}^{\nu}([g(a), g(b)]):=\left\{h \in C^{[\nu]}([g(a), g(b)]):\right. \\
\left.J_{1-\alpha}^{g\left(x_{0}\right)} h^{([\nu])} \in C^{1}\left(\left[g\left(x_{0}\right), g(b)\right]\right)\right\} .
\end{gathered}
$$

So let $h \in C_{g\left(x_{0}\right)}^{\nu}([g(a), g(b)])$; we define the left $g$-generalized fractional derivative of $h$ of order $\nu$, of Canavati type, over $\left[g\left(x_{0}\right), g(b)\right]$ as

$$
D_{g\left(x_{0}\right)}^{\nu} h:=\left(J_{1-\alpha}^{g\left(x_{0}\right)} h^{([\nu])}\right)^{\prime} .
$$


Clearly, for $h \in C_{g\left(x_{0}\right)}^{\nu}([g(a), g(b)])$, there exists

$$
\left(D_{g\left(x_{0}\right)}^{\nu} h\right)(z)=\frac{1}{\Gamma(1-\alpha)} \frac{d}{d z} \int_{g\left(x_{0}\right)}^{z}(z-t)^{-\alpha} h^{([\nu])}(t) d t
$$

for all $g\left(x_{0}\right) \leq z \leq g(b)$.

In particular, when $f \circ g^{-1} \in C_{g\left(x_{0}\right)}^{\nu}([g(a), g(b)])$ we have that

$$
\left(D_{g\left(x_{0}\right)}^{\nu}\left(f \circ g^{-1}\right)\right)(z)=\frac{1}{\Gamma(1-\alpha)} \frac{d}{d z} \int_{g\left(x_{0}\right)}^{z}(z-t)^{-\alpha}\left(f \circ g^{-1}\right)^{([\nu])}(t) d t
$$

for all $g\left(x_{0}\right) \leq z \leq g(b)$. We have

$$
D_{g\left(x_{0}\right)}^{n}\left(f \circ g^{-1}\right)=\left(f \circ g^{-1}\right)^{(n)} \text { and } D_{g\left(x_{0}\right)}^{0}\left(f \circ g^{-1}\right)=f \circ g^{-1} .
$$

We mention and need the following left generalized $g$-fractional, of Canavati type, Taylor's formula:

Theorem 3. Let $f \circ g^{-1} \in C_{g\left(x_{0}\right)}^{\nu}([g(a), g(b)])$, where $x_{0} \in[a, b]$ is fixed.

(i) if $\nu \geq 1$, then

$$
\begin{gathered}
f(x)-f\left(x_{0}\right)=\sum_{k=1}^{[\nu]-1} \frac{\left(f \circ g^{-1}\right)^{(k)}\left(g\left(x_{0}\right)\right)}{k !}\left(g(x)-g\left(x_{0}\right)\right)^{k} \\
+\frac{1}{\Gamma(\nu)} \int_{g\left(x_{0}\right)}^{g(x)}(g(x)-t)^{\nu-1}\left(D_{g\left(x_{0}\right)}^{\nu}\left(f \circ g^{-1}\right)\right)(t) d t
\end{gathered}
$$

all $x \in[a, b]: x \geq x_{0}$;

(ii) if $0<\nu<1$, we get

$$
f(x)=\frac{1}{\Gamma(\nu)} \int_{g\left(x_{0}\right)}^{g(x)}(g(x)-t)^{\nu-1}\left(D_{g\left(x_{0}\right)}^{\nu}\left(f \circ g^{-1}\right)\right)(t) d t,
$$

all $x \in[a, b]: x \geq x_{0}$.

By the change of variables method, see [10], we may rewrite the remainder of (16), (17), as

$$
\frac{1}{\Gamma(\nu)} \int_{g\left(x_{0}\right)}^{g(x)}(g(x)-t)^{\nu-1}\left(D_{g\left(x_{0}\right)}^{\nu}\left(f \circ g^{-1}\right)\right)(t) d t
$$




$$
=\frac{1}{\Gamma(\nu)} \int_{x_{0}}^{x}(g(x)-g(s))^{\nu-1}\left(D_{g\left(x_{0}\right)}^{\nu}\left(f \circ g^{-1}\right)\right)(g(s)) g^{\prime}(s) d s,
$$

all $x \in[a, b]: x \geq x_{0}$.

We may rewrite (17) as follows: if $0<\nu<1$, we have

$$
f(x)=\left(J_{\nu}^{g\left(x_{0}\right)}\left(D_{g\left(x_{0}\right)}^{\nu}\left(f \circ g^{-1}\right)\right)\right)(g(x)),
$$

all $x \in[a, b]: x \geq x_{0}$.

II) Next we follow [3], pp. 345-348.

Let $h \in C([g(a), g(b)])$, we define the right Riemann-Liouville fractional integral as

$$
\left(J_{z_{0}-}^{\nu} h\right)(z):=\frac{1}{\Gamma(\nu)} \int_{z}^{z_{0}}(t-z)^{\nu-1} h(t) d t,
$$

for $g(a) \leq z \leq z_{0} \leq g(b)$. We set $J_{z_{0}-}^{0} h=h$.

Let $\alpha:=\nu-[\nu](0<\alpha<1)$. We define the subspace $C_{g\left(x_{0}\right)-}^{\nu}([g(a)$, $g(b)])$ of $C^{[\nu]}([g(a), g(b)])$, where $x_{0} \in[a, b]$ :

$$
\begin{gathered}
C_{g\left(x_{0}\right)-}^{\nu}([g(a), g(b)]) \\
:=\left\{h \in C^{[\nu]}([g(a), g(b)]): J_{g\left(x_{0}\right)-}^{1-\alpha} h^{([\nu])} \in C^{1}\left(\left[g(a), g\left(x_{0}\right)\right]\right)\right\} .
\end{gathered}
$$

So let $h \in C_{g\left(x_{0}\right)-}^{\nu}([g(a), g(b)])$; we define the right $g$-generalized fractional derivative of $h$ of order $\nu$, of Canavati type, over $\left[g(a), g\left(x_{0}\right)\right]$ as

$$
D_{g\left(x_{0}\right)-}^{\nu} h:=(-1)^{n-1}\left(J_{g\left(x_{0}\right)-}^{1-\alpha} h^{([\nu])}\right)^{\prime} .
$$

Clearly, for $h \in C_{g\left(x_{0}\right)-}^{\nu}([g(a), g(b)])$, there exists

$$
\left(D_{g\left(x_{0}\right)-}^{\nu} h\right)(z)=\frac{(-1)^{n-1}}{\Gamma(1-\alpha)} \frac{d}{d z} \int_{z}^{g\left(x_{0}\right)}(t-z)^{-\alpha} h^{([\nu])}(t) d t
$$

for all $g(a) \leq z \leq g\left(x_{0}\right) \leq g(b)$.

In particular, when $f \circ g^{-1} \in C_{g\left(x_{0}\right)-}^{\nu}([g(a), g(b)])$ we have that

$$
\left(D_{g\left(x_{0}\right)-}^{\nu}\left(f \circ g^{-1}\right)\right)(z)=\frac{(-1)^{n-1}}{\Gamma(1-\alpha)} \frac{d}{d z} \int_{z}^{g\left(x_{0}\right)}(t-z)^{-\alpha}\left(f \circ g^{-1}\right)^{([\nu])}(t) d t
$$

for all $g(a) \leq z \leq g\left(x_{0}\right) \leq g(b)$. 
We get that

$$
\left(D_{g\left(x_{0}\right)-}^{n}\left(f \circ g^{-1}\right)\right)(z)=(-1)^{n}\left(f \circ g^{-1}\right)^{(n)}(z)
$$

and $\left(D_{g\left(x_{0}\right)-}^{0}\left(f \circ g^{-1}\right)\right)(z)=\left(f \circ g^{-1}\right)(z)$, all $z \in\left[g(a), g\left(x_{0}\right)\right]$.

We mention and need the following right generalized $g$-fractional, of Canavati type, Taylor's formula:

Theorem 4. Let $f \circ g^{-1} \in C_{g\left(x_{0}\right)-}^{\nu}([g(a), g(b)])$, where $x_{0} \in[a, b]$ is fixed.

(i) if $\nu \geq 1$, then

$$
\begin{gathered}
f(x)-f\left(x_{0}\right)=\sum_{k=1}^{[\nu]-1} \frac{\left(f \circ g^{-1}\right)^{(k)}\left(g\left(x_{0}\right)\right)}{k !}\left(g(x)-g\left(x_{0}\right)\right)^{k} \\
+\frac{1}{\Gamma(\nu)} \int_{g(x)}^{g\left(x_{0}\right)}(t-g(x))^{\nu-1}\left(D_{g\left(x_{0}\right)-}^{\nu}\left(f \circ g^{-1}\right)\right)(t) d t
\end{gathered}
$$

all $a \leq x \leq x_{0}$,

(ii) if $0<\nu<1$, we get

$$
f(x)=\frac{1}{\Gamma(\nu)} \int_{g(x)}^{g\left(x_{0}\right)}(t-g(x))^{\nu-1}\left(D_{g\left(x_{0}\right)-}^{\nu}\left(f \circ g^{-1}\right)\right)(t) d t
$$

all $a \leq x \leq x_{0}$

By change of variable, see [10], we may rewrite the remainder of $(26),(27)$, as

$$
\begin{gathered}
\frac{1}{\Gamma(\nu)} \int_{g(x)}^{g\left(x_{0}\right)}(t-g(x))^{\nu-1}\left(D_{g\left(x_{0}\right)-}^{\nu}\left(f \circ g^{-1}\right)\right)(t) d t \\
=\frac{1}{\Gamma(\nu)} \int_{x}^{x_{0}}(g(s)-g(x))^{\nu-1}\left(D_{g\left(x_{0}\right)-}^{\nu}\left(f \circ g^{-1}\right)\right)(g(s)) g^{\prime}(s) d s,
\end{gathered}
$$

all $a \leq x \leq x_{0}$.

We may rewrite (27) as follows:

if $0<\nu<1$, we have

$$
f(x)=\left(J_{g\left(x_{0}\right)-}^{\nu}\left(D_{g\left(x_{0}\right)-}^{\nu}\left(f \circ g^{-1}\right)\right)\right)(g(x)),
$$

all $a \leq x \leq x_{0} \leq b$. 
III) Denote by

$$
D_{g\left(x_{0}\right)}^{m \nu}=D_{g\left(x_{0}\right)}^{\nu} D_{g\left(x_{0}\right)}^{\nu} \cdots D_{g\left(x_{0}\right)}^{\nu} \quad(m \text {-times }), m \in \mathbb{N} .
$$

Also denote by

$$
J_{m \nu}^{g\left(x_{0}\right)}=J_{\nu}^{g\left(x_{0}\right)} J_{\nu}^{g\left(x_{0}\right)} \ldots J_{\nu}^{g\left(x_{0}\right)} \quad(m \text {-times }), m \in \mathbb{N} .
$$

We call $D_{g\left(x_{0}\right)}^{m \nu}$ an iterated fractional derivative.

We mention and need the following $g$-modified and generalized left fractional Taylor's formula of Canavati type:

Theorem 5. Let $0<\nu<1$. Assume that $\left(D_{g\left(x_{0}\right)}^{i \nu}\left(f \circ g^{-1}\right)\right) \in$ $C_{g\left(x_{0}\right)}^{\nu}([g(a), g(b)]), x_{0} \in[a, b]$, for $i=0,1, \ldots, m$. Assume also that $\left(D_{g\left(x_{0}\right)}^{(m+1) \nu}\left(f \circ g^{-1}\right)\right) \in C\left(\left[g\left(x_{0}\right), g(b)\right]\right)$. Then,

$$
\begin{gathered}
f(x)=\frac{1}{\Gamma((m+1) \nu)} \int_{g\left(x_{0}\right)}^{g(x)}(g(x)-z)^{(m+1) \nu-1}\left(D_{g\left(x_{0}\right)}^{(m+1) \nu}\left(f \circ g^{-1}\right)\right)(z) d z \\
=\frac{1}{\Gamma((m+1) \nu)} \int_{x_{0}}^{x}(g(x)-g(s))^{(m+1) \nu-1} \\
\quad \times\left(D_{g\left(x_{0}\right)}^{(m+1) \nu}\left(f \circ g^{-1}\right)\right)(g(s)) g^{\prime}(s) d s,
\end{gathered}
$$

all $x_{0} \leq x \leq b$

IV) Denote by

$$
D_{g\left(x_{0}\right)-}^{m \nu}=D_{g\left(x_{0}\right)-}^{\nu} D_{g\left(x_{0}\right)-}^{\nu} \ldots D_{g\left(x_{0}\right)-}^{\nu}(m \text {-times }), m \in \mathbb{N} \text {. }
$$

Also denote by

$$
J_{g\left(x_{0}\right)-}^{m \nu}=J_{g\left(x_{0}\right)-}^{\nu} J_{g\left(x_{0}\right)-\cdots}^{\nu} J_{g\left(x_{0}\right)-}^{\nu}(m \text {-times }), m \in \mathbb{N} \text {. }
$$

We call $D_{g\left(x_{0}\right) \text { - }}^{m \nu}$ an iterated fractional derivative.

We mention and need the following $g$-modified and generalized right fractional Taylor's formula of Canavati type: 
Theorem 6. Let $0<\nu<1$. Assume that $\left(D_{g\left(x_{0}\right)-}^{i \nu}\left(f \circ g^{-1}\right)\right) \in$ $C_{g\left(x_{0}\right)-}^{\nu}([g(a), g(b)]), x_{0} \in[a, b]$, for all $i=0,1, \ldots, m$. Assume also that $\left(D_{g\left(x_{0}\right)-}^{(m+1) \nu}\left(f \circ g^{-1}\right)\right) \in C\left(\left[g(a), g\left(x_{0}\right)\right]\right)$. Then,

$$
\begin{aligned}
f(x)=\frac{1}{\Gamma((m+1) \nu)} \int_{g(x)}^{g\left(x_{0}\right)}(z-g(x))^{(m+1) \nu-1}\left(D_{g\left(x_{0}\right)-}^{(m+1) \nu}\left(f \circ g^{-1}\right)\right)(z) d z \\
=\frac{1}{\Gamma((m+1) \nu)} \int_{x}^{x_{0}}(g(s)-g(x))^{(m+1) \nu-1} \\
\times\left(D_{g\left(x_{0}\right)-}^{(m+1) \nu}\left(f \circ g^{-1}\right)\right)(g(s)) g^{\prime}(s) d s
\end{aligned}
$$

all $a \leq x \leq x_{0} \leq b$.

In what follows next it is based on this background.

\section{Main results}

We present the following generalized $g$-fractional Polya type integral inequalities without any boundary conditions.

Theorem 7. Let $0<\nu<1, f \in C([a, b]), g:[a, b] \rightarrow \mathbb{R}$ be strictly increasing and $g \in C^{1}([a, b])$. Assume

and

$$
f \circ g^{-1} \in C_{g(a)}^{\nu}\left(\left[g(a), \frac{g(a)+g(b)}{2}\right]\right),
$$

Set

$$
f \circ g^{-1} \in C_{g(b)-}^{\nu}\left(\left[\frac{g(a)+g(b)}{2}, g(b)\right]\right) \text {. }
$$

$$
\begin{gathered}
M(f, g):=\max \left\{\left\|D_{g(a)}^{\nu}\left(f \circ g^{-1}\right)\right\|_{\infty,\left[g(a), \frac{g(a)+g(b)}{2}\right]},\right. \\
\left.\left\|D_{g(b)-}^{\nu}\left(f \circ g^{-1}\right)\right\|_{\infty,\left[\frac{g(a)+g(b)}{2}, g(b)\right]}\right\} .
\end{gathered}
$$

Then

$$
\left|\int_{a}^{b} f d g\right| \leq \int_{a}^{b}|f| d g \leq M(f, g) \frac{(g(b)-g(a))^{\nu+1}}{\Gamma(\nu+2) 2^{\nu}} .
$$

Inequality (37) is sharp, namely it is attained by $f_{*}$ such that 


$$
\left(f_{*} \circ g^{-1}\right)(z)=\left\{\begin{array}{cl}
(z-g(a))^{\nu}, & z \in\left[g(a), \frac{g(a)+g(b)}{2}\right] \\
(g(b)-z)^{\nu}, & z \in\left[\frac{g(a)+g(b)}{2}, g(b)\right]
\end{array}\right\}, \quad 0<\nu<1 .
$$

Clearly here non zero constant functions $f \circ g^{-1}$ are excluded.

Proof. Notice that

$$
\left|\int_{a}^{b} f d g\right|=\left|\int_{a}^{b} f g^{\prime} d x\right| \leq \int_{a}^{b}|f| g^{\prime} d x=\int_{a}^{b}|f| d g .
$$

Let $x_{0} \in[a, b]$ be such that $g\left(x_{0}\right)=\frac{g(a)+g(b)}{2}$, that is $x_{0}=g^{-1}\left(\frac{g(a)+g(b)}{2}\right)$. that

Let $f \circ g^{-1} \in C_{g(a)}^{\nu}\left(\left[g(a), \frac{g(a)+g(b)}{2}\right]\right), 0<\nu<1$. By Theorem 3 we have

$$
f(x)=\frac{1}{\Gamma(\nu)} \int_{g(a)}^{g(x)}(g(x)-t)^{\nu-1}\left(D_{g(a)}^{\nu}\left(f \circ g^{-1}\right)\right)(t) d t
$$

all $x \in\left[a, x_{0}\right]$. that

Assume also $f \circ g^{-1} \in C_{g(b)-}^{\nu}\left(\left[\frac{g(a)+g(b)}{2}, g(b)\right]\right)$, by Theorem 4 we have

$$
f(x)=\frac{1}{\Gamma(\nu)} \int_{g(x)}^{g(b)}(t-g(x))^{\nu-1}\left(D_{g(b)-}^{\nu}\left(f \circ g^{-1}\right)\right)(t) d t,
$$

all $x \in\left[x_{0}, b\right]$.

By (39) we get

$$
\begin{gathered}
|f(x)| \leq \frac{1}{\Gamma(\nu)}\left(\int_{g(a)}^{g(x)}(g(x)-t)^{\nu-1} d t\right)\left\|D_{g(a)}^{\nu}\left(f \circ g^{-1}\right)\right\|_{\infty,\left[g(a), \frac{g(a)+g(b)}{2}\right]} \\
=\left\|D_{g(a)}^{\nu}\left(f \circ g^{-1}\right)\right\|_{\infty,\left[g(a), \frac{g(a)+g(b)}{2}\right]} \frac{(g(x)-g(a))^{\nu}}{\Gamma(\nu+1)} .
\end{gathered}
$$

That is,

$$
|f(x)| \leq\left\|D_{g(a)}^{\nu}\left(f \circ g^{-1}\right)\right\|_{\infty,\left[g(a), \frac{g(a)+g(b)}{2}\right]} \frac{(g(x)-g(a))^{\nu}}{\Gamma(\nu+1)},
$$

for any $x \in\left[a, x_{0}\right]$.

Similarly, by (40) we get

$$
|f(x)| \leq \frac{1}{\Gamma(\nu)}\left(\int_{g(x)}^{g(b)}(t-g(x))^{\nu-1} d t\right)\left\|D_{g(b)-}^{\nu}\left(f \circ g^{-1}\right)\right\|_{\infty,\left[\frac{g(a)+g(b)}{2}, g(b)\right]}
$$




$$
=\frac{(g(b)-g(x))^{\nu}}{\Gamma(\nu+1)}\left\|D_{g(b)-}^{\nu}\left(f \circ g^{-1}\right)\right\|_{\infty,\left[\frac{g(a)+g(b)}{2}, g(b)\right]} .
$$

That is

$$
|f(x)| \leq\left\|D_{g(b)-}^{\nu}\left(f \circ g^{-1}\right)\right\|_{\infty,\left[\frac{g(a)+g(b)}{2}, g(b)\right]} \frac{(g(b)-g(x))^{\nu}}{\Gamma(\nu+1)}
$$

for any $x \in\left[x_{0}, b\right]$.

Thus, we have

$$
\begin{aligned}
& \left|\int_{a}^{b} f d g\right| \leq \int_{a}^{b}|f| d g=\int_{a}^{b}|f| g^{\prime} d x \\
& =\int_{a}^{x_{0}}|f(x)| g^{\prime}(x) d x+\int_{x_{0}}^{b}|f(x)| g^{\prime}(x) d x \stackrel{\text { (by (42), (44)) }}{\leq} \\
& \frac{\left\|D_{g(a)}^{\nu}\left(f \circ g^{-1}\right)\right\|_{\infty,\left[g(a), \frac{g(a)+g(b)}{2}\right]}}{\Gamma(\nu+1)} \int_{a}^{x_{0}}(g(x)-g(a))^{\nu} g^{\prime}(x) d x \\
& +\frac{\left\|D_{g(b)-}^{\nu}\left(f \circ g^{-1}\right)\right\|_{\infty,\left[\frac{g(a)+g(b)}{2}, g(b)\right]}}{\Gamma(\nu+1)} \int_{x_{0}}^{b}(g(b)-g(x))^{\nu} g^{\prime}(x) d x \\
& =\frac{\left\|D_{g(a)}^{\nu}\left(f \circ g^{-1}\right)\right\|_{\infty,\left[g(a), \frac{g(a)+g(b)}{2}\right]}}{\Gamma(\nu+1)} \frac{\left(g\left(x_{0}\right)-g(a)\right)^{\nu+1}}{(\nu+1)} \\
& +\frac{\left\|D_{g(b)-}^{\nu}\left(f \circ g^{-1}\right)\right\|_{\infty,\left[\frac{g(a)+g(b)}{2}, g(b)\right]}}{\Gamma(\nu+1)} \frac{\left(g(b)-g\left(x_{0}\right)\right)^{\nu+1}}{(\nu+1)} \\
& =\frac{1}{\Gamma(\nu+2)}\left[\left\|D_{g(a)}^{\nu}\left(f \circ g^{-1}\right)\right\|_{\infty,\left[g(a), \frac{g(a)+g(b)}{2}\right]}\left(\frac{g(b)-g(a)}{2}\right)^{\nu+1}\right. \\
& \left.+\left\|D_{g(b)-}^{\nu}\left(f \circ g^{-1}\right)\right\|_{\infty,\left[\frac{g(a)+g(b)}{2}, g(b)\right]}\left(\frac{g(b)-g(a)}{2}\right)^{\nu+1}\right] \\
& =\frac{1}{\Gamma(\nu+2)} \frac{\left(g(b)-g(a)^{\nu+1}\right)}{2^{\nu+1}}\left[\left\|D_{g(a)}^{\nu}\left(f \circ g^{-1}\right)\right\|_{\infty,\left[g(a), \frac{g(a)+g(b)}{2}\right]}\right. \\
& \left.+\left\|D_{g(b)-}^{\nu}\left(f \circ g^{-1}\right)\right\|_{\infty,\left[\frac{g(a)+g(b)}{2}, g(b)\right]}\right]
\end{aligned}
$$




$$
\leq \frac{M(f, g)}{\Gamma(\nu+2)} \frac{(g(b)-g(a))^{\nu+1}}{2^{\nu}} .
$$

We have proved inequality (37). Next we prove sharpness of (37).

Here $[\nu]=0$ and $\alpha=\nu$. We see that

$$
\begin{gathered}
\left(J_{1-\alpha}^{g(a)}\left(f_{*} \circ g^{-1}\right)\right)(z)=\frac{1}{\Gamma(1-\alpha)} \int_{g(a)}^{z}(z-t)^{-\alpha}\left(f_{*} \circ g^{-1}\right)(t) d t \\
=\frac{1}{\Gamma(1-\alpha)} \int_{g(a)}^{z}(z-t)^{(1-\alpha)-1}(t-g(a))^{(\nu+1)-1} d t
\end{gathered}
$$

(by [16], p. 256)

$$
=\frac{1}{\Gamma(1-\alpha)} \frac{\Gamma(1-\alpha) \Gamma(\nu+1)}{\Gamma(2)}(z-g(a))=\Gamma(\nu+1)(z-g(a)) .
$$

Therefore,

$$
\left(D_{g(a)}^{\nu}\left(f_{*} \circ g^{-1}\right)\right)(z)=\Gamma(\nu+1), \text { for all } z \in\left[g(a), \frac{g(a)+g(b)}{2}\right] .
$$

That is,

$$
\left\|D_{g(a)}^{\nu}\left(f \circ g^{-1}\right)\right\|_{\infty,\left[g(a), \frac{g(a)+g(b)}{2}\right]}=\Gamma(\nu+1) .
$$

Furthermore we have

$$
\begin{gathered}
-\left(J_{g(b)-}^{1-\alpha}\left(f_{*} \circ g^{-1}\right)\right)(z)=\frac{-1}{\Gamma(1-\alpha)} \int_{z}^{g(b)}(t-z)^{-\alpha}(g(b)-t)^{\nu} d t \\
=-\frac{1}{\Gamma(1-\alpha)} \int_{z}^{g(b)}(g(b)-t)^{(\nu+1)-1}(t-z)^{(1-\alpha)-1} d t
\end{gathered}
$$

(by [16], p. 256)

$$
=-\frac{1}{\Gamma(1-\alpha)} \frac{\Gamma(\nu+1) \Gamma(1-\alpha)}{\Gamma(2)}(g(b)-z)=\Gamma(\nu+1)(z-g(b)),
$$

for all $z \in\left[\frac{g(a)+g(b)}{2}, g(b)\right]$.

Hence

$$
\left(D_{g(b)-}^{\nu}\left(f_{*} \circ g^{-1}\right)\right)(z)=\Gamma(\nu+1),
$$

and

$$
\left\|D_{g(b)-}^{\nu}\left(f_{e} \circ g^{-1}\right)\right\|_{\infty,\left[\frac{g(a)+g(b)}{2}, g(b)\right]}=\Gamma(\nu+1) .
$$


Consequently we get that

$$
M\left(f_{*}, g\right)=\Gamma(\nu+1)
$$

Applying $f_{*}$ into (37) we obtain:

$$
\text { R.H.S (37) for } f_{*}=\frac{\Gamma(\nu+1)}{\Gamma(\nu+2)} \frac{(g(b)-g(a))^{\nu+1}}{2^{\nu}}=\frac{(g(b)-g(a))^{\nu+1}}{(\nu+1) 2^{\nu}} .
$$

We get the same result when we apply $f_{*}$ in the

$$
\begin{gathered}
\text { L.H.S }(37)=\left|\int_{a}^{b} f_{*}(x) d g(x)\right| \\
=\left|\int_{a}^{x_{0}} f_{*}(x) d g(x)+\int_{x_{0}}^{b} f_{*}(x) d g(x)\right| \\
=\left|\int_{a}^{x_{0}}\left(f_{*} \circ g^{-1}\right)(g(x)) d g(x)+\int_{x_{0}}^{b}\left(f_{*} \circ g^{-1}\right)(g(x)) d g(x)\right| \\
=\left|\int_{g(a)}^{g\left(x_{0}\right)}\left(f_{*} \circ g^{-1}\right)(z) d z+\int_{g\left(x_{0}\right)}^{g(b)}\left(f_{*} \circ g^{-1}\right)(z) d z\right| \\
=\left|\int_{g(a)}^{g\left(x_{0}\right)}(z-g(a))^{\nu} d z+\int_{g\left(x_{0}\right)}^{g(b)}(g(b)-z)^{\nu} d z\right| \\
=\frac{\left(g\left(x_{0}\right)-g(a)\right)^{\nu+1}}{\nu+1}+\frac{\left(g(b)-g\left(x_{0}\right)\right)^{\nu+1}}{\nu+1} \\
=\frac{2}{\nu+1} \frac{(g(b)-g(a))^{\nu+1}}{2^{\nu+1}}=\frac{(g(b)-g(a))^{\nu+1}}{(\nu+1) 2^{\nu}} .
\end{gathered}
$$

So equality holds in $(37)$ for $f_{*}$. Furthermore we notice that

$$
\begin{gathered}
\left(f_{*} \circ g^{-1}\right)\left(\left(\frac{g(a)+g(b)}{2}\right)_{-}\right)=\left(f_{*} \circ g^{-1}\right)\left(\left(\frac{g(a)+g(b)}{2}\right)_{+}\right) \\
=\left(\frac{g(b)-g(a)}{2}\right)^{\nu},
\end{gathered}
$$

thus $f_{*} \circ g^{-1}$ is continuous on $[g(a), g(b)]$ and hence $f_{*} \in C([a, b])$.

Sharpness of (37) has been proved. The theorem is proved completely. 
Remark 8. When $\nu \geq 1$, thus $n=[\nu] \geq 1$, and by assuming that $\left(f \circ g^{-1}\right)^{(k)}(g(a))=\left(f \circ g^{-1}\right)^{(k)}(g(b))=0$, for $k=0,1, \ldots, n-1$, we can prove inequality (37) again. Here also $f \in C^{n}([a, b])$ and $g^{-1} \in C^{n}([g(a), g(b)])$.

The function $\left(f \circ g^{-1}\right)^{(n)}$ cannot be a constant different than zero, equivalently, $f \circ g^{-1}$ cannot be a non-trivial polynomial of degree $n$.

We continue with a fractional iterated Polya type inequality:

Theorem 9. Let $0<\nu<1, f \in C([a, b]), g:[a, b] \rightarrow \mathbb{R}$ be strictly increasing and $g \in C^{1}([a, b])$. Assume that

$$
\left(D_{g(a)}^{i \nu}\left(f \circ g^{-1}\right)\right) \in C_{g(a)}^{\nu}\left(\left[g(a), \frac{g(a)+g(b)}{2}\right]\right),
$$

for $i=0,1, \ldots, m \in \mathbb{N}$, and $\left(D_{g(a)}^{(m+1) \nu}\left(f \circ g^{-1}\right)\right) \in C\left(\left[g(a), \frac{g(a)+g(b)}{2}\right]\right)$. Also assume that

$$
\left(D_{g(b)-}^{i \nu}\left(f \circ g^{-1}\right)\right) \in C_{g(b)-}^{\nu}\left(\left[\frac{g(a)+g(b)}{2}, g(b)\right]\right),
$$

for $i=0,1, \ldots, m \in \mathbb{N}$, and

$$
\left(D_{g(b)-}^{(m+1) \nu}\left(f \circ g^{-1}\right)\right) \in C\left(\left[\frac{g(a)+g(b)}{2}, g(b)\right]\right) \text {. }
$$

Set

$$
\begin{gathered}
M^{*}(f, g):=\max \left\{\left\|D_{g(a)}^{(m+1) \nu}\left(f \circ g^{-1}\right)\right\|_{\infty,\left[g(a), \frac{g(a)+g(b)}{2}\right]}\right. \\
\left.\left\|D_{g(b)-}^{(m+1) \nu}\left(f \circ g^{-1}\right)\right\|_{\infty,\left[\frac{g(a)+g(b)}{2}, g(b)\right]}\right\}
\end{gathered}
$$

Then

$$
\left|\int_{a}^{b} f d g\right| \leq \int_{a}^{b}|f| d g \leq M^{*}(f, g) \frac{(g(b)-g(a))^{(m+1) \nu+1}}{\Gamma((m+1) \nu+2) 2^{(m+1) \nu}}
$$

Proof. By Theorem 5 we have

$$
\begin{aligned}
f(x)= & \frac{1}{\Gamma((m+1) \nu)} \int_{g(a)}^{g(x)}(g(x)-z)^{(m+1) \nu-1} \\
& \times\left(D_{g(a)}^{(m+1) \nu}\left(f \circ g^{-1}\right)\right)(z) d z
\end{aligned}
$$


all $x \in\left[a, x_{0}\right]$, where $x_{0}:=g^{-1}\left(\frac{g(a)+g(b)}{2}\right)$.

By Theorem 6 we have

$$
\begin{aligned}
f(x)= & \frac{1}{\Gamma((m+1) \nu)} \int_{g(x)}^{g(b)}(z-g(x))^{(m+1) \nu-1} \\
& \times\left(D_{g(b)-}^{(m+1) \nu}\left(f \circ g^{-1}\right)\right)(z) d z
\end{aligned}
$$

all $x \in\left[x_{0}, b\right]$.

By (62) we get

$$
\begin{gathered}
|f(x)| \leq \frac{1}{\Gamma((m+1) \nu)} \\
\left(\int_{g(a)}^{g(x)}(g(x)-z)^{(m+1) \nu-1} d z\right)\left\|D_{g(a)}^{(m+1) \nu}\left(f \circ g^{-1}\right)\right\|_{\infty,\left[g(a), \frac{g(a)+g(b)}{2}\right]} \\
=\frac{(g(x)-g(a))^{(m+1) \nu}}{\Gamma((m+1) \nu+1)}\left\|D_{g(a)}^{(m+1) \nu}\left(f \circ g^{-1}\right)\right\|_{\infty,\left[g(a), \frac{g(a)+g(b)}{2}\right]}
\end{gathered}
$$

for all $x \in\left[a, x_{0}\right]$.

Similarly, by (63), we obtain

$$
\begin{gathered}
|f(x)| \leq \frac{1}{\Gamma((m+1) \nu)} \\
\left(\int_{g(x)}^{g(b)}(z-g(x))^{(m+1) \nu-1} d z\right)\left\|D_{g(b)-}^{(m+1) \nu}\left(f \circ g^{-1}\right)\right\|_{\infty,\left[\frac{g(a)+g(b)}{2}, g(b)\right]} \\
=\frac{(g(b)-g(x))^{(m+1) \nu}}{\Gamma((m+1) \nu+1)}\left\|D_{g(b)-}^{(m+1) \nu}\left(f \circ g^{-1}\right)\right\|_{\infty,\left[\frac{g(a)+g(b)}{2}, g(b)\right]},
\end{gathered}
$$

for all $x \in\left[x_{0}, b\right]$.

Thus, we have

$$
\begin{gathered}
\left|\int_{a}^{b} f d g\right| \leq \int_{a}^{b}|f| d g=\int_{a}^{b}|f| g^{\prime} d x \\
=\int_{a}^{x_{0}}|f(x)| g^{\prime}(x) d x+\int_{x_{0}}^{b}|f(x)| g^{\prime}(x) d x \text { (by (64), (65)) } \\
\leq \frac{\left\|D_{g(a)}^{(m+1) \nu}\left(f \circ g^{-1}\right)\right\|_{\infty,\left[g(a), \frac{g(a)+g(b)}{2}\right]}}{\Gamma((m+1) \nu+1)} \int_{a}^{x_{0}}(g(x)-g(a))^{(m+1) \nu} g^{\prime}(x) d x \\
+\frac{\left\|D_{g(b)-}^{(m+1) \nu}\left(f \circ g^{-1}\right)\right\|_{\infty,\left[\frac{g(a)+g(b)}{2}, g(b)\right]}}{\Gamma((m+1) \nu+1)} \int_{x_{0}}^{b}(g(b)-g(x))^{(m+1) \nu} g^{\prime}(x) d x
\end{gathered}
$$




$$
\begin{gathered}
=\frac{1}{\Gamma((m+1) \nu+2)} \\
\times\left[\left\|D_{g(a)}^{(m+1) \nu}\left(f \circ g^{-1}\right)\right\|_{\infty,\left[g(a), \frac{g(a)+g(b)}{2}\right]}\left(g\left(x_{0}\right)-g(a)\right)^{(m+1) \nu+1}\right. \\
\left.+\left\|D_{g(b)-}^{(m+1) \nu}\left(f \circ g^{-1}\right)\right\|_{\infty,\left[\frac{g(a)+g(b)}{2}, g(b)\right]}\left(g(b)-g\left(x_{0}\right)\right)^{(m+1) \nu+1]}\right]
\end{gathered}
$$

(by $\left.g\left(x_{0}\right)=\frac{g(a)+g(b)}{2}\right)$

$$
\begin{aligned}
= & \frac{1}{\Gamma((m+1) \nu+2)} \frac{(g(b)-g(a))^{(m+1) \nu+1}}{2^{(m+1) \nu+1}} \\
& {\left[\left\|D_{g(a)}^{(m+1) \nu}\left(f \circ g^{-1}\right)\right\|_{\infty,\left[g(a), \frac{g(a)+g(b)}{2}\right]}\right.} \\
& \left.+\left\|D_{g(b)-}^{(m+1) \nu}\left(f \circ g^{-1}\right)\right\|_{\infty,\left[\frac{g(a)+g(b)}{2}, g(b)\right]}\right] \\
\leq & \frac{M^{*}(f, g)}{\Gamma((m+1) \nu+2)} \frac{(g(b)-g(a))^{(m+1) \nu+1}}{2^{(m+1) \nu}},
\end{aligned}
$$

proving inequality (61).

Next we give an $L_{1}$ generalized $g$-fractional Polya inequality:

Theorem 10. Let $\nu \geq 1, n=[\nu]$, and $f \in C^{n}([a, b]), g:[a, b] \rightarrow$ $\mathbb{R}$ be a strictly increasing function. Assume that $g \in C^{1}([a, b])$ and $g^{-1} \in$ $C^{n}([g(a), g(b)])$. Also assume $f \circ g^{-1} \in C_{g(a)}^{\nu}\left(\left[g(a), \frac{g(a)+g(b)}{2}\right]\right)$, and $f \circ g^{-1} \in$ $C_{g(b)-}^{\nu}\left(\left[\frac{g(a)+g(b)}{2}, g(b)\right]\right)$, and that $\left(f \circ g^{-1}\right)^{(k)}(g(a))=\left(f \circ g^{-1}\right)^{(k)}(g(b))=$ 0 , for $k=0,1, \ldots, n-1$. Set

$$
\begin{gathered}
M_{1}(f, g):=\max \left\{\left\|D_{g(a)}^{\nu}\left(f \circ g^{-1}\right)\right\|_{L_{1}\left(\left[g(a), \frac{g(a)+g(b)}{2}\right]\right)},\right. \\
\left.\left\|D_{g(b)-}^{\nu}\left(f \circ g^{-1}\right)\right\|_{L_{1}\left(\left[\frac{g(a)+g(b)}{2}, g(b)\right]\right)}\right\} .
\end{gathered}
$$

Then

$$
\left|\int_{a}^{b} f d g\right| \leq \int_{a}^{b}|f| d g \leq M_{1}(f, g) \frac{(g(b)-g(a))^{\nu}}{\Gamma(\nu+1) 2^{\nu-1}} .
$$

Here $f \circ g^{-1}$ cannot be a non-trivial polynomial of degree $n$. 
Proof. Let $f \circ g^{-1} \in C_{g(a)}^{\nu}\left(\left[g(a), \frac{g(a)+g(b)}{2}\right]\right), \quad \nu \geq 1$, and $\left(f \circ g^{-1}\right)^{(k)}(g(a))=0$, for $k=0,1, \ldots, n-1, n=[\nu]$. By Theorem 3 we have that

$$
f(x)=\frac{1}{\Gamma(\nu)} \int_{g(a)}^{g(x)}(g(x)-t)^{\nu-1}\left(D_{g(a)}^{\nu}\left(f \circ g^{-1}\right)\right)(t) d t,
$$

all $x \in\left[a, x_{0}\right]$, where $x_{0}:=g^{-1}\left(\frac{g(a)+g(b)}{2}\right)$.

Let $f \circ g^{-1} \in C_{g(b)-}^{\nu}\left(\left[\frac{g(a)+g(b)}{2}, g(b)\right]\right)$, and $\left(f \circ g^{-1}\right)^{(k)}(g(b))=0$, for $k=0,1, \ldots, n-1$. By Theorem 4 we have that

$$
f(x)=\frac{1}{\Gamma(\nu)} \int_{g(x)}^{g(b)}(t-g(x))^{\nu-1}\left(D_{g(b)-}^{\nu}\left(f \circ g^{-1}\right)\right)(t) d t,
$$

all $x \in\left[x_{0}, b\right]$.

By (71) we have

$$
\begin{gathered}
|f(x)| \leq \frac{1}{\Gamma(\nu)} \int_{g(a)}^{g(x)}(g(x)-t)^{\nu-1}\left|\left(D_{g(a)}^{\nu}\left(f \circ g^{-1}\right)\right)(t)\right| d t \leq \\
\quad \frac{(g(x)-g(a))^{\nu-1}}{\Gamma(\nu)} \int_{g(a)}^{g(x)}\left|\left(D_{g(a)}^{\nu}\left(f \circ g^{-1}\right)\right)(t)\right| d t \leq \\
\frac{(g(x)-g(a))^{\nu-1}}{\Gamma(\nu)}\left\|D_{g(a)}^{\nu}\left(f \circ g^{-1}\right)\right\|_{L_{1}\left(\left[g(a), \frac{g(a)+g(b)}{2}\right]\right)},
\end{gathered}
$$

for all $x \in\left[a, x_{0}\right]$.

Similarly, by (72) we get

$$
\begin{gathered}
|f(x)| \leq \frac{1}{\Gamma(\nu)} \int_{g(x)}^{g(b)}(t-g(x))^{\nu-1}\left|\left(D_{g(b)-}^{\nu}\left(f \circ g^{-1}\right)\right)(t)\right| d t \leq \\
\frac{(g(b)-g(x))^{\nu-1}}{\Gamma(\nu)} \int_{g(x)}^{g(b)}\left|\left(D_{g(b)-}^{\nu}\left(f \circ g^{-1}\right)\right)(t)\right| d t \leq \\
\frac{(g(b)-g(x))^{\nu-1}}{\Gamma(\nu)}\left\|D_{g(b)-}^{\nu}\left(f \circ g^{-1}\right)\right\|_{L_{1}\left(\left[\frac{g(a)+g(b)}{2}, g(b)\right]\right)},
\end{gathered}
$$

for all $x \in\left[x_{0}, b\right]$.

Thus, we have

$$
\begin{gathered}
\left|\int_{a}^{b} f d g\right| \leq \int_{a}^{b}|f| d g=\int_{a}^{b}|f| g^{\prime} d x \\
=\int_{a}^{x_{0}}|f(x)| g^{\prime}(x) d x+\int_{x_{0}}^{b}|f(x)| g^{\prime}(x) d x \stackrel{\text { (by (73), (74)) }}{\leq}
\end{gathered}
$$




$$
\begin{aligned}
& \frac{\left\|D_{g(a)}^{\nu}\left(f \circ g^{-1}\right)\right\|_{L_{1}\left(\left[g(a), \frac{g(a)+g(b)}{2}\right]\right)}}{\Gamma(\nu)} \int_{a}^{x_{0}}(g(x)-g(a))^{\nu-1} g^{\prime}(x) d x \\
& +\frac{\left\|D_{g(b)-}^{\nu}\left(f \circ g^{-1}\right)\right\|_{L_{1}\left(\left[\frac{g(a)+g(b)}{2}, g(b)\right]\right)}}{\Gamma(\nu)} \int_{x_{0}}^{b}(g(b)-g(x))^{\nu-1} g^{\prime}(x) d x \\
& =\frac{\left\|D_{g(a)}^{\nu}\left(f \circ g^{-1}\right)\right\|_{L_{1}\left(\left[g(a), \frac{g(a)+g(b)}{2}\right]\right)}}{\Gamma(\nu+1)}\left(g\left(x_{0}\right)-g(a)\right)^{\nu} \\
& +\frac{\left\|D_{g(b)-}^{\nu}\left(f \circ g^{-1}\right)\right\|_{L_{1}\left(\left[\frac{g(a)+g(b)}{2}, g(b)\right]\right)}}{\Gamma(\nu+1)}\left(g(b)-g\left(x_{0}\right)\right)^{\nu} \\
& =\frac{1}{\Gamma(\nu+1)} \frac{(g(b)-g(a))^{\nu}}{2^{\nu}}\left[\left\|D_{g(a)}^{\nu}\left(f \circ g^{-1}\right)\right\|_{L_{1}\left(\left[g(a), \frac{g(a)+g(b)}{2}\right]\right)}\right. \\
& \left.+\left\|D_{g(b)-}^{\nu}\left(f \circ g^{-1}\right)\right\|_{L_{1}\left(\left[\frac{g(a)+g(b)}{2}, g(b)\right]\right)}\right] \\
& \leq \frac{M_{1}(f, g)}{\Gamma(\nu+1)} \frac{(g(b)-g(a))^{\nu}}{2^{\nu-1}}
\end{aligned}
$$

proving the claim.

We continue with a $L_{q}$ generalized $g$-fractional Polya type inequality:

Theorem 11. Let $p, q>1: \frac{1}{p}+\frac{1}{q}=1, \nu>\frac{1}{q}, n=[\nu], f \in C^{n}([a, b])$, $g:[a, b] \rightarrow \mathbb{R}$ be a strictly increasing function. Assume that $g \in C^{1}([a, b])$ and $g^{-1} \in C^{n}([g(a), g(b)])$. Also assume $f \circ g^{-1} \in C_{g(a)}^{\nu}\left(\left[g(a), \frac{g(a)+g(b)}{2}\right]\right)$, and $f \circ$ $g^{-1} \in C_{g(b)-}^{\nu}\left(\left[\frac{g(a)+g(b)}{2}, g(b)\right]\right)$, and that $\left(f \circ g^{-1}\right)^{(k)}(g(a))=\left(f \circ g^{-1}\right)^{(k)}(g(b))=$ 0 , for $k=0,1, \ldots, n-1$. When $\frac{1}{q}<\nu<1$, the last boundary conditions are void. Set

$$
\begin{gathered}
M_{q}(f, g):=\max \left\{\left\|D_{g(a)}^{\nu}\left(f \circ g^{-1}\right)\right\|_{L_{q}}\left(\left[g(a), \frac{g(a)+g(b)}{2}\right]\right),\right. \\
\left.\left\|D_{g(b)-}^{\nu}\left(f \circ g^{-1}\right)\right\|_{L_{q}\left(\left[\frac{g(a)+g(b)}{2}, g(b)\right]\right)}\right\} .
\end{gathered}
$$

Then 


$$
\leq \frac{\left|\int_{a}^{b} f d g\right| \leq \int_{a}^{b}|f| d g}{2^{\nu-\frac{1}{q}} \Gamma(\nu)(p(\nu-1)+1)^{\frac{1}{p}}\left(\nu+\frac{1}{p}\right)}(g(b)-g(a))^{\nu+\frac{1}{p}} .
$$

Again here $f \circ g^{-1}$ cannot be a non-trivial polynomial of degree $n$.

Proof. Let $f \circ g^{-1} \in C_{g(a)}^{\nu}\left(\left[g(a), \frac{g(a)+g(b)}{2}\right]\right), \nu>0, \quad$ and $\left(f \circ g^{-1}\right)^{(k)}(g(a))=0$, for $k=0,1, \ldots, n-1, n=[\nu]$. By Theorem 3 we have that

$$
f(x)=\frac{1}{\Gamma(\nu)} \int_{g(a)}^{g(x)}(g(x)-t)^{\nu-1}\left(D_{g(a)}^{\nu}\left(f \circ g^{-1}\right)\right)(t) d t,
$$

all $x \in\left[a, x_{0}\right]$, where $x_{0}:=g^{-1}\left(\frac{g(a)+g(b)}{2}\right)$.

Let $f \circ g^{-1} \in C_{g(b)-}^{\nu}\left(\left[\frac{g(a)+g(b)}{2}, g(b)\right]\right)$, and $\left(f \circ g^{-1}\right)^{(k)}(g(b))=0$, for $k=0,1, \ldots, n-1$. By Theorem 4 we have that

$$
f(x)=\frac{1}{\Gamma(\nu)} \int_{g(x)}^{g(b)}(t-g(x))^{\nu-1}\left(D_{g(b)-}^{\nu}\left(f \circ g^{-1}\right)\right)(t) d t,
$$

all $x \in\left[x_{0}, b\right]$.

By (79) we have

$$
\begin{gathered}
|f(x)| \leq \frac{1}{\Gamma(\nu)} \int_{g(a)}^{g(x)}(g(x)-t)^{\nu-1}\left|\left(D_{g(a)}^{\nu}\left(f \circ g^{-1}\right)\right)(t)\right| d t \leq \\
\frac{1}{\Gamma(\nu)}\left(\int_{g(a)}^{g(x)}(g(x)-t)^{p(\nu-1)} d t\right)^{\frac{1}{p}}\left(\int_{g(a)}^{g(x)}\left|\left(D_{g(a)}^{\nu}\left(f \circ g^{-1}\right)\right)(t)\right|^{q} d t\right)^{\frac{1}{q}} \\
\leq \frac{1}{\Gamma(\nu)} \frac{(g(x)-g(a))^{\nu-1+\frac{1}{p}}}{(p(\nu-1)+1)^{\frac{1}{p}}}\left\|D_{g(a)}^{\nu}\left(f \circ g^{-1}\right)\right\|_{L_{q}\left(\left[g(a) \frac{g(a)+g(b)}{2}\right]\right)}
\end{gathered}
$$

for all $x \in\left[a, x_{0}\right]$.

Similarly, by (80) we get

$$
\begin{gathered}
|f(x)| \leq \frac{1}{\Gamma(\nu)} \int_{g(x)}^{g(b)}(t-g(x))^{\nu-1}\left|\left(D_{g(b)-}^{\nu}\left(f \circ g^{-1}\right)\right)(t)\right| d t \leq \\
\frac{1}{\Gamma(\nu)}\left(\int_{g(x)}^{g(b)}(t-g(x))^{p(\nu-1)} d t\right)^{\frac{1}{p}}\left(\int_{g(x)}^{g(b)}\left|\left(D_{g(b)-}^{\nu}\left(f \circ g^{-1}\right)\right)(t)\right|^{q} d t\right)^{\frac{1}{q}}
\end{gathered}
$$




$$
\leq \frac{1}{\Gamma(\nu)} \frac{(g(b)-g(x))^{\nu-1+\frac{1}{p}}}{(p(\nu-1)+1)^{\frac{1}{p}}}\left\|D_{g(b)-}^{\nu}\left(f \circ g^{-1}\right)\right\|_{L_{q}\left(\left[\frac{g(a)+g(b)}{2}, g(b)\right]\right)},
$$

for all $x \in\left[x_{0}, b\right]$.

Thus, we have

$$
\begin{aligned}
& \left|\int_{a}^{b} f d g\right| \leq \int_{a}^{b}|f| d g= \\
& \int_{a}^{x_{0}}|f(x)| g^{\prime}(x) d x+\int_{x_{0}}^{b}|f(x)| g^{\prime}(x) d x \stackrel{(\text { by }(81),(82))}{\leq} \frac{1}{\Gamma(\nu)(p(\nu-1)+1)^{\frac{1}{p}}} \\
& {\left[\left\|D_{g(a)}^{\nu}\left(f \circ g^{-1}\right)\right\|_{L_{q}\left(\left[g(a), \frac{g(a)+g(b)}{2}\right]\right)} \int_{a}^{x_{0}}(g(x)-g(a))^{\nu-1+\frac{1}{p}} g^{\prime}(x) d x\right.} \\
& \left.+\left\|D_{g(b)-}^{\nu}\left(f \circ g^{-1}\right)\right\|_{L_{q}\left(\left[\frac{g(a)+g(b)}{2}, g(b)\right]\right)} \int_{x_{0}}^{b}(g(b)-g(x))^{\nu-1+\frac{1}{p}} g^{\prime}(x) d x\right] \\
& =\frac{1}{\Gamma(\nu)(p(\nu-1)+1)^{\frac{1}{p}}\left(\nu+\frac{1}{p}\right)} \\
& \times\left[\left\|D_{g(a)}^{\nu}\left(f \circ g^{-1}\right)\right\|_{L_{q}\left(\left[g(a), \frac{g(a)+g(b)}{2}\right]\right)}\left(g\left(x_{0}\right)-g(a)\right)^{\nu+\frac{1}{p}}\right. \\
& \left.+\left\|D_{g(b)-}^{\nu}\left(f \circ g^{-1}\right)\right\|_{L_{q}\left(\left[\frac{g(a)+g(b)}{2}, g(b)\right]\right)}\left(g(b)-g\left(x_{0}\right)\right)^{\nu+\frac{1}{p}}\right] \\
& =\frac{1}{\Gamma(\nu)(p(\nu-1)+1)^{\frac{1}{p}}\left(\nu+\frac{1}{p}\right)} \frac{(g(b)-g(a))^{\nu+\frac{1}{p}}}{2^{\nu+\frac{1}{p}}} \times \\
& {\left[\left\|D_{g(a)}^{\nu}\left(f \circ g^{-1}\right)\right\|_{L_{q}\left(\left[g(a), \frac{g(a)+g(b)}{2}\right]\right)}+\left\|D_{g(b)-}^{\nu}\left(f \circ g^{-1}\right)\right\|_{L_{q}\left(\left[\frac{g(a)+g(b)}{2}, g(b)\right]\right)}\right]} \\
& \leq \frac{M_{q}(f, g)}{\Gamma(\nu)(p(\nu-1)+1)^{\frac{1}{p}}\left(\nu+\frac{1}{p}\right)} \frac{(g(b)-g(a))^{\nu+\frac{1}{p}}}{2^{\nu-\frac{1}{q}}}
\end{aligned}
$$

proving the claim.

Combining facts from Theorems 7, 10, 11 and Remark 8, we get: 
Theorem 12. Let $p, q>1: \frac{1}{p}+\frac{1}{q}=1, \nu \geq 1, n=[\nu], f \in C^{n}([a, b])$, $g:[a, b] \rightarrow \mathbb{R}$ be a strictly increasing function. Assume that $g \in C^{1}([a, b])$ and $g^{-1} \in C^{n}([g(a), g(b)])$. Also assume $f \circ g^{-1} \in C_{g(a)}^{\nu}\left(\left[g(a), \frac{g(a)+g(b)}{2}\right]\right)$, and $f \circ g^{-1} \in C_{g(b)-}^{\nu}\left(\left[\frac{g(a)+g(b)}{2}, g(b)\right]\right)$, and that

$$
\left(f \circ g^{-1}\right)^{(k)}(g(a))=\left(f \circ g^{-1}\right)^{(k)}(g(b))=0,
$$

for $k=0,1, \ldots, n-1$. Then

$$
\begin{gathered}
\left|\int_{a}^{b} f d g\right| \leq \int_{a}^{b}|f| d g \\
\leq \min \left\{M(f, g) \frac{(g(b)-g(a))^{\nu+1}}{\Gamma(\nu+2) 2^{\nu}}, M_{1}(f, g) \frac{(g(b)-g(a))^{\nu}}{\Gamma(\nu+1) 2^{\nu-1}},\right. \\
\left.\frac{M_{q}(f, g)}{2^{\nu-\frac{1}{q}} \Gamma(\nu)(p(\nu-1)+1)^{\frac{1}{p}}\left(\nu+\frac{1}{p}\right)}(g(b)-g(a))^{\nu+\frac{1}{p}}\right\},
\end{gathered}
$$

where $M(f, g)$ as in (36), $M_{1}(f, g)$ as in (69) and $M_{q}(f, g)$ as in (77). Above $f \circ g^{-1}$ cannot be a non-trivial polynomial of degree $n$.

Corollary 13. Here all as in Theorem 12. Then

$$
\begin{gathered}
\left|\frac{1}{g(b)-g(a)} \int_{a}^{b} f d g\right| \leq \frac{1}{g(b)-g(a)} \int_{a}^{b}|f| d g \\
\leq \min \left\{M(f, g) \frac{(g(b)-g(a))^{\nu}}{\Gamma(\nu+2) 2^{\nu}}, M_{1}(f, g) \frac{(g(b)-g(a))^{\nu-1}}{\Gamma(\nu+1) 2^{\nu-1}},\right. \\
\left.\frac{M_{q}(f, g)}{2^{\nu-\frac{1}{q}} \Gamma(\nu)(p(\nu-1)+1)^{\frac{1}{p}}\left(\nu+\frac{1}{p}\right)}(g(b)-g(a))^{\nu-\frac{1}{q}}\right\} .
\end{gathered}
$$

We continue with an $L_{1}$ iterated fractional Polya type inequality:

Theorem 14. All as in Theorem 9, with $\frac{1}{m+1} \leq \nu<1$. Set

$$
M_{1}^{*}(f, g):=\max \left\{\left\|D_{g(a)}^{(m+1) \nu}\left(f \circ g^{-1}\right)\right\|_{L_{1}\left(\left[g(a), \frac{g(a)+g(b)}{2}\right]\right)},\right.
$$




$$
\left.\left\|D_{g(b)-}^{(m+1) \nu}\left(f \circ g^{-1}\right)\right\|_{L_{1}\left(\left[\frac{g(a)+g(b)}{2}, g(b)\right]\right)}\right\}
$$

Then

$$
\left|\int_{a}^{b} f d g\right| \leq \int_{a}^{b}|f| d g \leq M_{1}^{*}(f, g) \frac{(g(b)-g(a))^{(m+1) \nu}}{\Gamma((m+1) \nu+1) 2^{(m+1) \nu-1}}
$$

Proof. Similar to Theorem 10.

We continue with an $L_{p}$ iterated fractional Polya type inequality:

Theorem 15. All as in Theorem 9. Let $p, q>1: \frac{1}{p}+\frac{1}{q}=1$, such that $\frac{1}{(m+1) q}<\nu<1$. Set

$$
\begin{gathered}
M_{q}^{*}(f, g):=\max \left\{\left\|D_{g(a)}^{(m+1) \nu}\left(f \circ g^{-1}\right)\right\|_{L_{q}\left(\left[g(a), \frac{g(a)+g(b)}{2}\right]\right)}\right. \\
\left.\left\|D_{g(b)-}^{(m+1) \nu}\left(f \circ g^{-1}\right)\right\|_{L_{q}\left(\left[\frac{g(a)+g(b)}{2}, g(b)\right]\right)}\right\}
\end{gathered}
$$

Then

$$
\begin{gathered}
\left|\int_{a}^{b} f d g\right| \leq \int_{a}^{b}|f| d g \leq \\
\frac{M_{q}^{*}(f, g)(g(b)-g(a))^{(m+1) \nu+\frac{1}{p}}}{2^{(m+1) \nu-\frac{1}{q}} \Gamma((m+1) \nu)(p((m+1) \nu-1)+1)^{\frac{1}{p}}\left((m+1) \nu+\frac{1}{p}\right)}
\end{gathered}
$$

Proof. Similar to Theorem 11.

Applications follow:

Proposition 16. Let $0<\nu<1, f \in C([a, b])$. Assume $f \circ \ln x \in$ $C_{e^{a}}^{\nu}\left(\left[e^{a}, \frac{e^{a}+e^{b}}{2}\right]\right)$, and $f \circ \ln x \in C_{\xi^{b}-}^{\nu}\left(\left[\frac{e^{a}+e^{b}}{2}, e^{b}\right]\right)$. Set

$$
\begin{gathered}
M\left(f, e^{x}\right):=\max \left\{\left\|D_{e^{a}}^{\nu}(f \circ \ln )\right\|_{\infty,\left[e^{a}, \frac{e^{a}+e^{b}}{2}\right]},\right. \\
\left.\left\|D_{e^{b}-}^{\nu}(f \circ \ln )\right\|_{\infty,\left[\frac{e^{a}+e^{b}}{2}, e^{b}\right]}\right\} .
\end{gathered}
$$

Then

$$
\left|\int_{a}^{b} f(x) e^{x} d x\right| \leq \int_{a}^{b}|f(x)| e^{x} d x \leq M\left(f, e^{x}\right) \frac{\left(e^{b}-e^{a}\right)^{\nu+1}}{\Gamma(\nu+2) 2^{\nu}}
$$


Inequality (92) is sharp, namely it is attained by $f_{*}$ such that

$$
\left(f_{*} \circ \ln x\right)(z)=\left\{\begin{array}{l}
\left(z-e^{a}\right)^{\nu}, \quad z \in\left[e^{a}, \frac{e^{a}+e^{b}}{2}\right] \\
\left(e^{b}-z\right)^{\nu}, z \in\left[\frac{e^{a}+e^{b}}{2}, e^{b}\right]
\end{array}\right\}, \quad 0<\nu<1 .
$$

Clearly here non zero constant functions $f \circ \ln x$ are excluded.

Proof. By Theorem 7.

We continue with

Proposition 17. Here $[a, b] \subset(0,+\infty), 0<\nu<1, f \in C([a, b])$. Assume that $\left(D_{\ln a}^{i \nu}\left(f \circ e^{x}\right)\right) \in C_{\ln a}^{\nu}\left(\left[\ln a, \frac{\ln (a b)}{2}\right]\right)$, for $i=0,1, \ldots, m \in \mathbb{N}$, and $\left(D_{\ln a}^{(m+1) \nu}\left(f \circ e^{x}\right)\right) \in C\left(\left[\ln a,\left(\frac{\ln a b}{2}\right)\right]\right)$. Also assume that $\left(D_{\ln b-}^{i \nu}\left(f \circ e^{x}\right)\right) \in$ $C_{\ln b-}^{\nu}\left(\left[\frac{\ln (a b)}{2}, \ln b\right]\right)$, for $i=0,1, \ldots, m \in \mathbb{N}$, and $\left(D_{\ln b-}^{(m+1) \nu}\left(f \circ e^{x}\right)\right) \in$ $C\left(\left[\frac{\ln (a b)}{2}, \ln b\right]\right)$. Set

$$
\begin{gathered}
M^{*}(f, \ln x):=\max \left\{\left\|D_{\ln a}^{(m+1) \nu}\left(f \circ e^{x}\right)\right\|_{\infty,\left[\ln a, \frac{\ln (a b)}{2}\right]},\right. \\
\left\|D_{\ln b-}^{(m+1) \nu}\left(f \circ e^{x}\right)\right\|_{\left.\infty,\left[\frac{\ln (a b)}{2}, \ln b\right]\right\} .}
\end{gathered}
$$

Then

$$
\left|\int_{a}^{b} \frac{f(x)}{x} d x\right| \leq \int_{a}^{b} \frac{|f(x)|}{x} d x \leq M^{*}(f, \ln x) \frac{\left(\ln \frac{b}{a}\right)^{(m+1) \nu+1}}{\Gamma((m+1) \nu+2) 2^{(m+1) \nu}} .
$$

Proof. By Theorem 9.

We can have many other interesting applications but we stop here.

\section{References}

[1] G.A. Anastassiou, Fractional Differentiation Inequalities, Research Monograph, Springer, New York, 2009.

[2] G.A. Anastassiou, On right fractional calculus, Chaos, Solitons and Fractals, 42 (2009), 365-376. 
[3] G.A. Anastassiou, Inteligent Mathematics: Computational Analysis, Springer, Heidelberg, 2011.

[4] G.A. Anastassiou, Intelligent Comparisons: Analytic Inequalities, Springer, Heidelberg - New York, 2016.

[5] G.A. Anastassiou, Generalized Canavati type fractional Taylor's formulae, J. Computational Analysis and Applications, 21, No 7 (2016), 1205-1212.

[6] J.A. Canavati, The Riemann-Liouville integral, Nieuw Archief Voor Wiskunde, 5, No 1 (1987), 53-75.

[7] A.M.A. El-Sayed, M. Gaber, On the finite Caputo and finite Riesz derivatives, Electronic J. of Theoretical Physics, 3, No 12 (2006), 81-95.

[8] G.S. Frederico, D.F.M. Torres, Fractional optimal control in the sense of Caputo and the fractional Noether's theorem, Internat. Math. Forum, 3, No 10 (2008), 479-493.

[9] R. Gorenflo, F. Mainardi, Essentials of Fractional Calculus, 2000, Maphysto Center, http://www.maphysto.dk/oldpages/events/LevyCAC2000/ MainardiNotes/fm2k0a.ps.

[10] Rong-Qing Jia, Chapter 3. Absolutely Continuous Functions, https://www.ualberta.ca/ rjia/Math418/Notes/Chap.3.pdf.

[11] G. Polya, Ein mittelwertsats für Funktionen mehrerer Veränderlichen, Tohoku Math. J., 19 (1921), 1-3.

[12] G. Polya and G. Szegö, Aufgaben und Lehrsärtze aus der Analysis, Volume I, Springer-Verlag, Berlin, 1925 (In German).

[13] G. Polya and G. Szegö, Problems and Theorems in Analysis, Volume I, Classics in Mathematics, Springer-Verlag, Berlin, 1972.

[14] G. Polya and G. Szegö, Problems and Theorems in Analysis, Volume I, Chinese Edition, 1984.

[15] S.G. Samko, A.A. Kilbas, O.I. Marichev, Fractional Integrals and Derivatives, Theory and Applications, Gordon and Breach, Amsterdam, 1993 [English transl. from the Russian, Integrals and Derivatives of Fractional Order and Some of Their Applications, Nauka i Tekhnika, Minsk, 1987]. 
[16] E.T. Whittaker and G.N. Watson, A Course in Modern Analysis, Cambridge University Press, 1927. 